Discussion Paper No. 08-055

\title{
Wie kann die durchschnittliche Ausbildungsquote in Deutschland korrekt gemessen werden?
}

Peter Jacobebbinghaus, Jens Mohrenweiser und Thomas Zwick

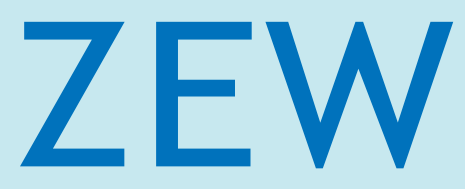

Zentrum für Europäische Wirtschaftsforschung $\mathrm{GmbH}$

Centre for European

Economic Research 


\title{
Wie kann die durchschnittliche Ausbildungsquote in Deutschland korrekt gemessen werden?
}

\author{
Peter Jacobebbinghaus, Jens Mohrenweiser \\ und Thomas Zwick
}

Download this ZEW Discussion Paper from our ftp server:

ftp://ftp.zew.de/pub/zew-docs/dp/dp08055.pdf

Die Discussion Papers dienen einer möglichst schnellen Verbreitung von neueren Forschungsarbeiten des ZEW. Die Beiträge liegen in alleiniger Verantwortung der Autoren und stellen nicht notwendigerweise die Meinung des ZEW dar.

Discussion Papers are intended to make results of ZEW research promptly available to other economists in order to encourage discussion and suggestions for revisions. The authors are solely responsible for the contents which do not necessarily represent the opinion of the ZEW. 


\section{Nichttechnische Zusammenfassung}

In den politischen Diskussionen über die Ursachen der Lehrstellenlücke in Deutschland während der letzten Jahre wurde häufig eine zu geringe Ausbildungsneigung der Unternehmen thematisiert, die sich in einer zu geringen Ausbildungsquote niederschlage.

In diesem Beitrag wird gezeigt, dass Lehrstellenlücke und Ausbildungsquote nur bedingt miteinander zusammenhängen. Die Lehrstellenlücke kann aufgrund größerer Kohorten, die auf den Arbeitsmarkt drängen sowie gesunkener Beschäftigungszahlen steigen, während die Ausbildungsquote gleich bleibt oder sogar steigt.

Außerdem wird thematisiert, dass die bisher gängige Berechnung der Ausbildungsquote an Stichtagen zu stark abweichenden Ergebnissen führt. Dies liegt daran, dass die Ausbildungsquote zyklisch über das Jahr schwankt mit einem Maximum zum Jahreswechsel und einem Minimum im Juli. Im Maximum liegt die Ausbildungsquote circa einen Prozentpunkt oberhalb des Durchschnitts und im Minimum circa einen halben Prozentpunkt darunter. Hinzu kommt, dass die saisonalen Muster zwischen den Berufsgruppen abweichen, da manche Berufe Abschlussprüfungen am Ende des Ausbildungsjahres vorsehen und andere Berufe nach dem Halbjahr. Dies bedeutet wiederum, dass die Abweichungen vom Jahresmittelwert für einzelne Berufe je nach Messzeitpunkt unterschiedlich sind.

Um die durchschnittliche Ausbildungsquote in Deutschland korrekt zu berechnen, schlagen wir eine Aggregation der täglich erhobenen Ausbildungsquoten vor. Die Beschäftigtenstatistik der Bundesagentur für Arbeit ist dabei die beste Datenbasis für die Berechnung der täglichen Ausbildungsquote. Der Beitrag zeigt, dass deutliche Verzerrungen auftreten können, wenn man die Ausbildungsquote auf Basis anderer Datenquellen berechnet. 


\section{Nontechnical Summary}

The political debate in Germany frequently blames shortcomings in the motivation of establishments to train apprentices for the undersupply of vacancies for dual apprenticeships. As a consequence it calls for an increase in the share of apprentices.

In this note we first show that the gap in dual apprenticeship vacancies and the share of apprentices are only weakly correlated. The apprenticeship gap may increase for example because larger cohorts enter the labour market or employment decreased while the apprenticeship share was constant or even increased.

Next, this paper shows that the usual way of calculating the share of apprentices on varying reference dates leads to a range of different estimates. The reason is that the share of apprentices fluctuates cyclically over the year. The maximum is around the turn of the year and the minimum is in July. At the maximum the share of apprentices is about one percent point higher than the yearly average and at the minimum the share of apprentices is about half a percent lower than the average.

An additional aspect is that the seasonal patterns deviate between occupational groups because some apprenticeships terminate at the end of the teaching term while others end at mid-term. As a consequence the deviations from the yearly average differ by occupation and they are dependent on the measurement point in time.

In order to properly calculate the average share of apprentices in Germany, we propose an aggregation of the daily share of apprentices to the yearly mean. We also argue that the employment statistics of the Federal Employment Agency provide the best data basis for calculating the daily share of apprentices. When other data sources are used to calculate the apprenticeship quote, large differences might occur. 


\title{
Wie kann die durchschnittliche Ausbildungsquote in Deutschland korrekt gemessen werden?*
}

\author{
Peter Jacobebbinghaus*, Jens Mohrenweiser und Thomas \\ Zwick ${ }^{\wedge}$ \\ * Forschungsdatenzentrum der Bundesagentur für Arbeit im IAB, Nürnberg \\ peter.jacobebbinghaus@iab.de \\ Universität Zürich, Institut für Strategie und Unternehmensökonomik, Zürich \\ jens.mohrenweiser@isu.uzh.ch \\ `Zentrum für Europäische Wirtschaftsforschung (ZEW), Mannheim \\ zwick@zew.de
}

August 2008

\begin{abstract}
This paper demonstrates that the share of apprentices exhibits a relatively strong seasonal pattern. This means that statistics on the share of apprentices such as those presented in official publications differ substantially from the actual yearly mean if they are measured on a date close to the minimum (end of July) or the maximum (around the turn of the year). In addition the deviation from the yearly mean differs between occupations at certain measurement dates because occupations have different apprenticeship durations. This paper proposes to calculate the yearly average on the basis of daily shares instead of certain measurement dates. It also argues to use daily accurate employment data from the German social security system.
\end{abstract}

JEL Codes: C81, J24

Key Words: Apprenticeship training, measurement

* Dieser Beitrag wurde finanziell durch das Projekt der Deutschen Forschungsgemeinschaft (DFG) „Heterogene Arbeit: Positive und normative Aspekte der Qualifikationsstruktur” sowie das Schweizer Bundesamt für Berufsbildung und Technologie durch ihr Leading House „Economics of Education, Firm Behaviour and Training Policies“ unterstützt. Wir danken Uschi Backes-Gellner, Stefan Bender, Hans Dietrich, Kathrin Göggel, Matthias Kräkel und Thomas Wachter für wichtige Anregungen. Die Datengrundlage dieses Beitrags bilden die schwach anonymisierte IAB-Beschäftigtenstichprobe (Version 1975-2004) und das Betriebs-Historik-Panel. Der Datenzugang erfolgte über einen Gastaufenthalt am Forschungsdatenzentrum der Bundesagentur für Arbeit im Institut für Arbeitsmarkt- und Berufsforschung (FDZ) und anschließend mittels kontrollierter Datenfernverarbeitung beim FDZ. 


\section{Einführung}

Es ist auffallend, dass in Deutschland keine amtlichen Daten über die Ausbildungsquote, d.h. den durchschnittlichen Anteil der Auszubildenden an allen Beschäftigten in den Unternehmen, erhoben werden. Die Ausbildungsquote hat aber weit reichende wirtschaftspolitische Konsequenzen. Nach Berufsbildungsgesetz ( $§ 86 \mathrm{BBiG}$ ), ist die Bundesregierung gehalten, sich jährlich zu vergewissern, ob das Angebots-Nachfrage-Verhältnis in der Berufsbildung politische Interventionen erforderlich machen könnte (Autorengruppe Bildungsberichterstattung, 2008). ${ }^{1}$ Um die in den letzten Jahren beobachtete Lehrstellenlücke zu verringern, droht die Politik beispielsweise regelmäßig mit einer Ausbildungsplatzabgabe. So wird im bisher nicht umgesetzten Entwurf des Berufsausbildungssicherungsgesetzes von 2004 im ersten Paragraph eine Ausbildungsumlagefinanzierung gefordert, wenn die Anzahl der freien Lehrstellen die Anzahl der Lehrstellenplatzsuchenden nicht um 15\% übersteigt (Wachter 2008). Die Kernannahme des Gesetzesentwurfes ist hierbei, dass mit einer festen einzelbetrieblichen Ausbildungsquote (hier: sieben Prozent) das Ziel der ausreichenden Versorgung mit Lehrstellen dauerhaft zu erreichen sei. Eine staatlich vorgegebene Ausbildungsquote scheint, wie weiter unten gezeigt, jedoch ungeeignet zu sein, um eine Lehrstellenlücken auf Dauer zu vermeiden, da die Ausbildungsquote kaum auf Schwankungen bei den Kohorten der Schulabgänger reagiert, während sie deutlich den zyklischen Schwankungen der Beschäftigung unterworfen ist. Nichtsdestotrotz ist es zunächst einmal notwendig, die Unterschiede der in der Literatur ausgewiesener Ausbildungsquoten zu erklären und einen Standard zur Berechnung zu finden, um die Ausbildungsquote festlegen zu können, die zum aktuellen Zeitpunkt die Lehrstellenlücke schließt.

Da keine amtliche Erhebung der Ausbildungsquote existiert, behelfen sich Publikationen über den Lehrlingsanteil in Betrieben mit Daten aus anderen Quellen, wie zum Beispiel den Befragungsdaten des IAB-Betriebspanels oder mit der IAB Beschäftigtenstichprobe. Bei einem Vergleich dieser Publikationen fällt sofort eine beachtliche Differenz der ausgewiesenen Ausbildungsquote im gleichen Jahr auf. So berechnet der Bericht „Bildung in Deutschland“ des Konsortiums Bildungsberichterstattung (2006, S. 88) eine Ausbildungs-

\footnotetext{
${ }^{1}$ Deshalb wird jährlich vom Bundesministerium für Bildung und Forschung der sogenannte Berufsbildungsbericht vorgelegt, der unter anderem das Angebot und die Nachfrage auf dem Ausbildungsstellenmarkt präsentiert. Da diese Größen von Erfassungsproblemen komplexer Marktprozesse geprägt sind, kommen hier restriktive Annahmen zum Einsatz, deren Realitätsnähe diskussionswürdig ist (Dietrich et al., 2008).
} 
quote von 6,4\% im Jahr 2004. Im Berufsbildungsbericht 2006 wird im Gegensatz dazu für das gleiche Jahr eine Ausbildungsquote von 4,8\% ausgewiesen (BMBF 2007, S. 144). In diesem Beitrag wird gezeigt, worin die Unterschiede in den jeweiligen Ausbildungsquoten begründet sind. Insbesondere zeigen wir, wie die durchschnittliche Ausbildungsquote in Deutschland berechnet werden kann. Dafür verwenden wir die IABBeschäftigtenstichprobe (IABS) und berechnen die Ausbildungsquote für jeden Tag des Jahres. Hierbei zeigt sich, dass die Ausbildungsquote erwartungsgemäß saisonal deutlich schwankt mit einem Maximum um den Jahreswechsel und einem Minimum im Sommer. Damit ist die über das Jahr berechnete Ausbildungsquote deutlich niedriger als beispielsweise bei Erhebungen am 31.12. wie im Bericht „Bildung in Deutschland“ und deutlich höher als am 30.6. wie im Berufsbildungsbericht. Eine Aufspaltung der Ausbildungsquote in unterschiedliche Berufsgruppen zeigt außerdem saisonal unterschiedlich schwankende Ausbildungsquoten in verschiedenen Berufsgruppen, abhängig von der üblichen Ausbildungslänge. Dadurch weichen die Ausbildungsquoten in den verschiedenen Berufsgruppen zu den einzelnen Messzeitpunkten unterschiedlich stark vom Mittelwert ab. Das gleiche Argument kann für den Nenner der Ausbildungsquote - der Beschäftigung gemacht werden. In manchen Berufen schwankt die Beschäftigung saisonal und ist deshalb zu bestimmten Zeitpunkten besonders niedrig (wie zum Beispiel im Baugewerbe oder in der Landwirtschaft im Winter).

Dieser Beitrag argumentiert somit, dass aufgrund der natürlichen und wiederkehrenden saisonalen Schwankungen die Aggregation täglicher Ausbildungsquoten auf Jahresdurchschnitte informativer ist als Zeitpunktbetrachtungen.

In den weiteren Abschnitten dieses Papiers wird zunächst die Entwicklung der Lehrstellenlücke, der Anzahl der sozialversicherungspflichtig Beschäftigten und der Ausbildungsquote zwischen 1999 und 2004 dargestellt (Abschnitt II). Nach der Gegenüberstellung der bekanntesten Messungen der Ausbildungsquote wird diskutiert, worin die unterschiedlichen Quoten begründet sind (Abschnitt III). Anschließend wird eine neue Methode zur korrekten Berechnung von Jahresmittelwerten vorgeschlagen, um die Nachteile der Stichtagswahl zu vermeiden (Abschnitt IV). Dann werden die Ergebnisse dieser tagesgenauen Messmethode für einzelne Berufsgruppen präsentiert (Abschnitt V). Der Beitrag endet mit Schlussfolgerungen. 


\section{Entwicklung der Lehrstellenlücke und der Ausbildungsquote in den letzten Jahren}

Zunächst grenzen wir die vieldiskutierten Begriffe der Ausbildungsquote und der Lehrstellenlücke voneinander ab. Die Ausbildungsquote ist der Anteil der Auszubildenden an allen Beschäftigten. Damit ist die Ausbildungsquote von der Entwicklung auf dem Ausbildungsstellenmarkt (Anzahl der Auszubildenden) und auf dem Arbeitsmarkt (Gesamtzahl der Beschäftigten) abhängig. Dagegen bezieht sich die Lehrstellenlücke auf die Differenz aus der Zahl der zur Verfügung stehenden Lehrstellen und der Zahl der Lehrstellenbewerber. Die Zahl der Lehrstellenbewerber enthält eine starke demografische Komponente. Deswegen kann die Lehrstellenlücke beispielsweise trotz einer gleich bleibenden Ausbildungsquote bei steigenden Schulabgängerzahlen oder einem steigenden Anteil von Schulabgängern, die an einer dualen Ausbildung interessiert sind, zunehmen.

Abbildung 1 zeigt die Veränderungen der Lehrstellenlücke von 2000 bis 2004. Die Zahl der Schulabgänger allgemeinbildender Schulen ist weitgehend konstant und steigt erst im Schuljahr 2003/2004 an. ${ }^{2}$ Trotzdem sinkt die Anzahl der Lehrstellenbewerber zunächst, da der Anteil der am dualen Ausbildungssystem interessierten Schulabgänger sinkt, und erst 2004 steigt die Anzahl der Lehrstellenbewerber wieder leicht an. ${ }^{3}$ Die von 2002 bis 2004 stark steigende Lehrstellenlücke ist somit vor allem auf sinkende Lehrstellenangebote und damit eine leicht sinkende Anzahl an Auszubildenden zurückzuführen. Das sinkende Lehrstellenangebot geht einher mit einer gesunkenen Gesamtbeschäftigung, denn die Ausbildungsquote blieb praktisch konstant.

Während die Anzahl der Schulabgänger und (mit Einschränkungen) die Ausbildungsquote aus amtlichen Statistiken berechnet werden können, basieren die Zahlen zur Lehrstellenlücke in Abbildung 1 auf freiwilligen Meldungen. Diese Angaben müssen sich jedoch nicht zwangsläufig mit den realen Größen decken und die reale Nachfrage und das reale Angebot kann somit je nach wirtschaftlicher Lage über- oder unterschätzt werden.

\footnotetext{
${ }^{2}$ Laut Institut der deutschen Wirtschaft (2007) und Ulrich et al. (2004) erhöhte sich die Gesamtzahl der Schulabgänger im Zeitraum 2000-2004 von 1,148 Mio. auf 1,256 Mio. Im Unterschied zu den Werten in Abbildung 1 sind dabei auch Absolventen mit an beruflichen Schulen erworbenen Abschlüssen eingerechnet. ${ }^{3}$ Der Anteil der dualen Ausbildung an den drei Systemen des beruflichen Ausbildungssystems sank im betrachteten Zeitraum von knapp 46\% auf gut 43\% (Konsortium Bildungsberichterstattung 2006, Tabelle E1.1 S. 80). Die anderen beiden beruflichen Ausbildungssysteme sind das Berufssystem in Vollzeitschulen und das so genannte Übergangssystem (zum Beispiel das schulische Berufsvorbereitungsjahr oder das schulische Berufsgrundbildungsjahr).
} 


\section{Abbildung 1: Schulabgänger, Ausbildungsbewerber, gemeldete Ausbildungsstellen Lehrstellenlücke und Ausbildungsquote im Oktober}

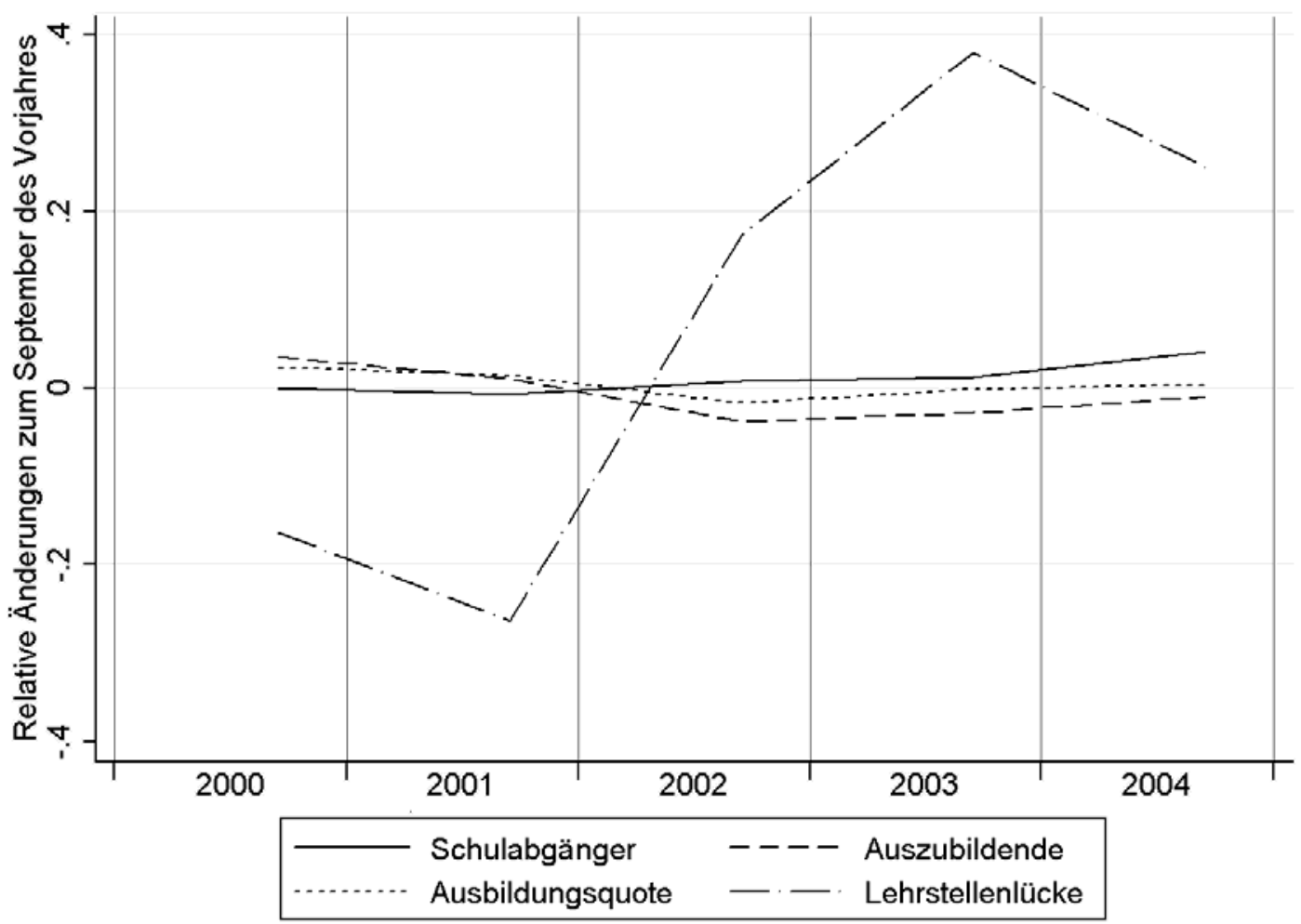

Anmerkungen: Stellen, Bewerber, Lücke und Ausbildungsquote beziehen sich auf den September. Die Zahl der die Schulabgänger bezieht sich auf das im selben Jahr abgelaufene Schuljahr und auf allgemeinbildende Schulen.

Quellen: Statistik der Bundesagentur für Arbeit (2007), Statistisches Bundesamt (2008), IABS 1975-2004, eigene Berechnungen.

Ebenfalls von der im Folgenden betrachteten Ausbildungsquote zu unterscheiden ist die durchschnittliche betriebliche Ausbildungsquote. Diese ergibt sich, indem die Ausbildungsquote zunächst auf Betriebsebene berechnet und dann über alle Betriebe gemittelt wird. Alle Betriebe gehen dabei mit dem gleichen Gewicht ein bzw. werden bei disproportionalen Stichproben mit ihrem Hochrechnungsfaktor gewichtet, nicht aber mit der Beschäftigtenzahl. Diese betriebsbezogene Betrachtung ist wenig aussagekräftig, da Aufspaltungen oder Zusammenschlüsse von Betrieben die Quote ändern können, obwohl der Anteil der Auszubildenden insgesamt dadurch unberührt bleibt. ${ }^{4}$

\footnotetext{
${ }^{4}$ Die durchschnittliche betriebliche Ausbildungsquote liegt etwa einen Prozentpunkt unter der Ausbildungsquote (siehe Abbildung 6 im Anhang). Das heißt, kleine Betriebe haben im Durchschnitt einen geringeren Anteil an Auszubildenden.
} 


\section{Abbildung 2: Sozialversicherungspflichtig Beschäftigte und Auszubildende zwischen}

1999 und 2004

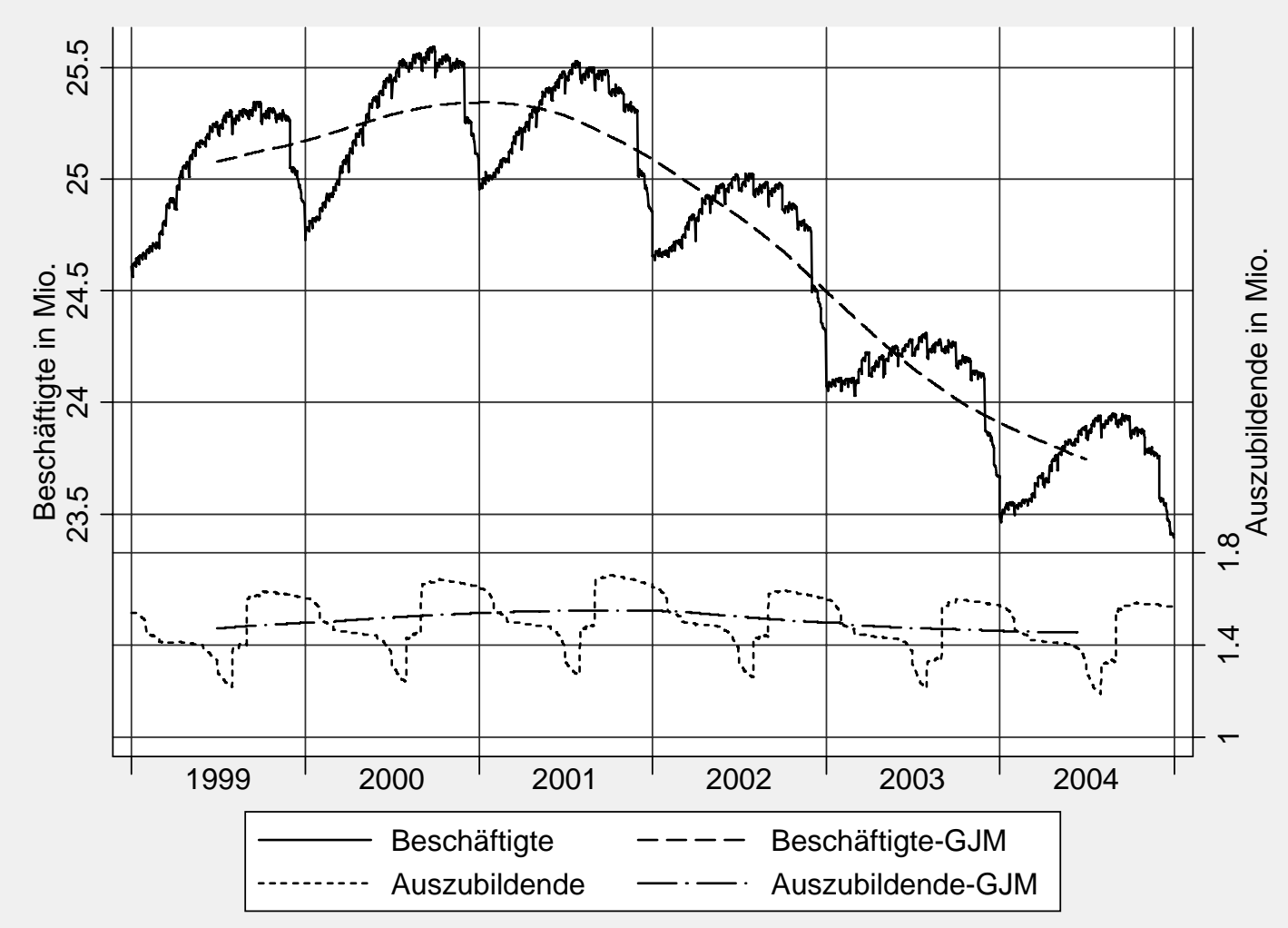

Anmerkungen: GJM steht für gleitender Jahresmittelwert.

Quelle: IABS 1975-2004, eigene Berechnungen.

Entscheidend für die Konstanz der Ausbildungsquote in den Jahren 1999 bis 2004 ist die Entwicklung der Zahl der sozialversicherungspflichtig Beschäftigten und der Auszubildenden, wie in Abbildung 2 dargestellt, und die daraus resultierende Ausbildungsquote (Abbildung 3). Die Werte basieren auf der IAB-Beschäftigtenstichprobe (1975-2004), die für eine 2-Prozent-Stichprobe der sozialversicherungspflichtig Beschäftigten in Deutschland tagesgenaue Angaben zu Beschäftigung und Leistungsbezug enthält (Drews 2007). Dadurch ist es möglich, zunächst die Ausbildungsquote für jeden Tag des betrachteten Zeitraums und anschließend - zur Verdeutlichung des Trends - den gleitenden Jahresmittelwert zu berechnen. $^{5}$

Das gleitende Jahresmittel der sozialversicherungspflichtig Beschäftigten steigt bis Januar 2001 an um danach zu sinken. Die Zahl der Auszubildenden erreicht sein Maximum etwas verzögert im August 2001. Die relative Änderung vom Maximum zum Minimum am Ende

${ }^{5}$ Zu den Details der Berechnung siehe Abschnitt IV und Fußnote 12. 
der Zeitreihe beträgt bei beiden Reihen etwa -6\%, was auf eine relativ konstante Ausbildungsquote hindeutet, sofern man die unterschiedlichen Saisonmuster herausrechnet. Abbildung 3 bestätigt dies: Das gleitende Jahresmittel der Ausbildungsquote steigt bis Januar 2002 auf 5,8\% und sinkt danach nur geringfügig. Interessant ist zudem, dass beispielsweise im Jahresvergleich zwischen 2003 und 2004 die Ausbildungsquote zum Messzeitpunkt 30.6. gefallen und zum Messzeitpunkt 31.12. gestiegen ist.

\section{Abbildung 3: Ausbildungsquote in Abhängigkeit vom Messzeitpunkt}

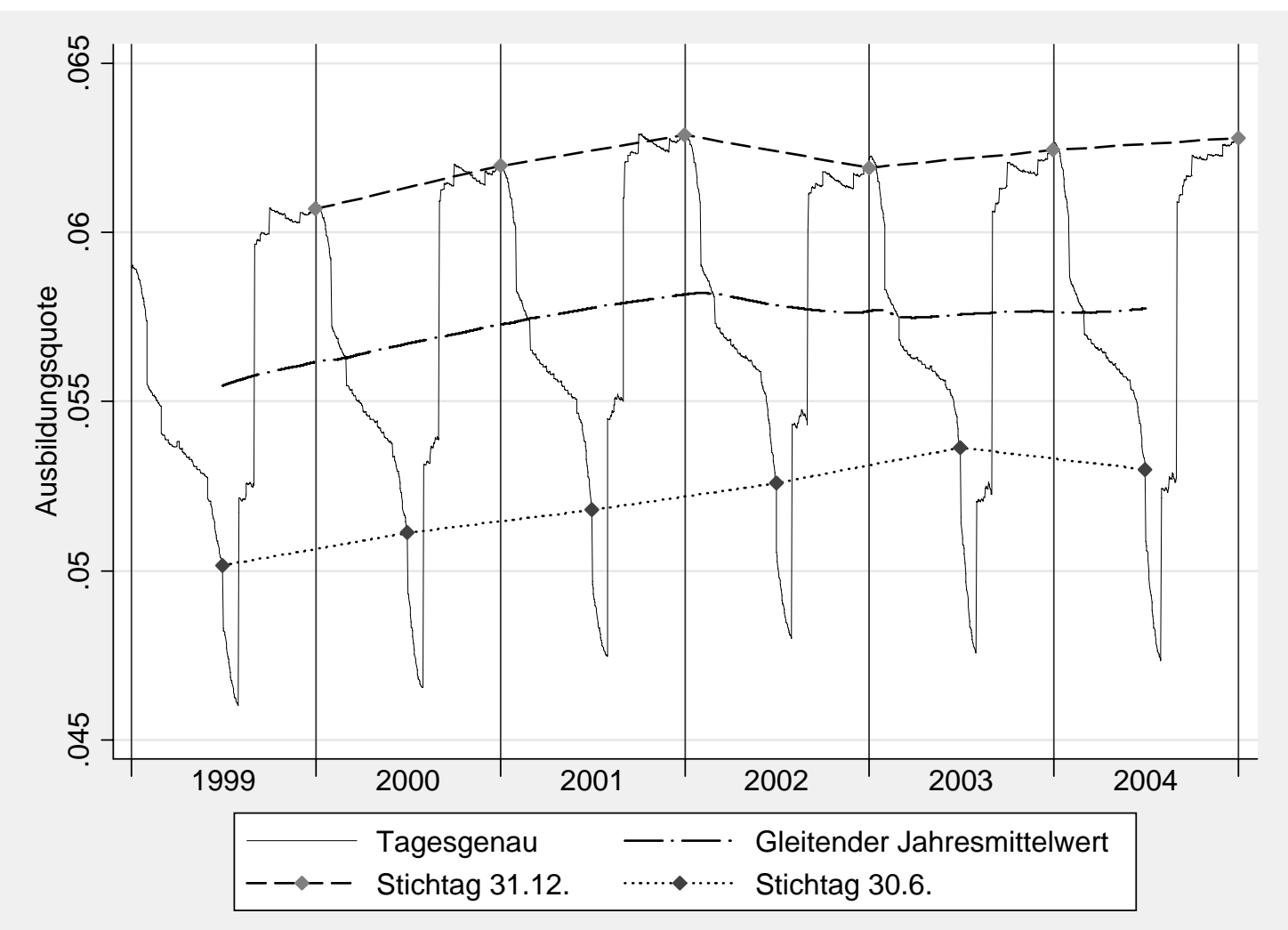

Quelle: IABS 1975-2004, eigene Berechnungen.

Dieser Abschnitt verdeutlicht, dass die absolute Anzahl der Ausbildungsplätze bei gleichbleibender oder sogar steigender Ausbildungsquote sinken kann, wenn die Anzahl der Beschäftigten stark genug abnimmt. ${ }^{6}$ Dieser Fall war zwischen den Jahren 2001 und 2004 zu beobachten. Dies kann bedeuten, dass in diesen Jahren bei einer gleichbleibenden Ausbildungsquote die Lehrstellenlücke steigt, wenn gleichzeitig mehr Schulabgänger eine duale

\footnotetext{
${ }^{6}$ Das Gegenteil war in den letzten Jahren der Fall - so stieg beispielsweise das Ausbildungsplatzangebot aufgrund der günstigen Beschäftigungsentwicklung auf dem Arbeitsmarkt zwischen 2006 und 2007 um 80.000 Stellen oder 14\%, während die Ausbildungsquote sich gemäß der Stichtagsberechnungen des Konsortiums Bildungsberichtserstattung (2008) nur leicht von 6.4 auf 6.5\% erhöhte.
} 
Ausbildung anstreben. Somit ist klar, dass mit einer festgelegten Ausbildungsquote Lehrstellenlücken nicht vermieden werden können.

\section{Ursachen der Unterschiede in Schätzungen der Ausbildungsquote}

Die ausgewiesene Ausbildungsquote weicht in den beiden wichtigsten Berichten zur Berufsbildung in Deutschland stark voneinander ab. Der Bericht Bildung in Deutschland weist auf der Basis der Beschäftigten- und Betriebsstatistik der BA zum Zeitpunkt 31.12. eine Ausbildungsquote von 6,4\% im Jahr 2000 aus, während der Berufsbildungsbericht, auf Basis des IAB-Betriebspanels, 4,9\% für das gleiche Jahr berechnet (Bundesministerium für Bildung und Forschung 2007). ${ }^{7}$ Neben diesen Publikationen an politische Entscheidungsträger weichen auch die Ausbildungsquoten zwischen wissenschaftlichen Publikationen stark voneinander ab, wobei die Spannweite von 4,93\% (Beckmann 2002, auf Grundlage des IAB-Betriebspanels im Durchschnitt 1993-2000) bis zu 8.09\% bei Dustmann und Schönberg (2004, LIAB 1996-1999) reicht. Für die unterschiedlichen Ausbildungsquoten gibt es eine Reihe von Ursachen, die wir im Folgenden erklären werden.

Zur Berechnung der Ausbildungsquote in Deutschland bieten sich im Wesentlichen vier Datenquellen an: die IAB Beschäftigtenstichprobe (Drews 2007), das IAB-Betriebspanel (Fischer et al. 2008), das Betriebs-Historik-Panel (Spengler 2007) und die BIBB-Erhebung "Neu abgeschlossene Ausbildungsverträge zum 30.09."8.

Die jeweiligen Messungen der Ausbildungsquote unterscheiden sich durch

- die zugrunde liegenden Datenquelle,

- den Bezugszeitpunkt,

- die Abgrenzung der Auszubildenden (Zähler) und der Beschäftigten (Nenner).

Alle betrachteten Datenquellen haben Stärken und Schwächen. Die IABS basiert auf den tagesgenauen Meldungen der Betriebe zur Sozialversicherung. Alle sozialversicherungsrelevanten Informationen sind zuverlässig. Ungenauigkeiten kann es bei ausschließlich zu statistischen Zwecken erhobenen Merkmalen geben, beispielsweise Beruf oder der Arbeitszeit. Dies ist zu beachten, wenn die Ausbildungsquote differenziert nach Personengruppen berechnet werden soll. Ausserdem ist der hohe Stichprobenumfang hervorzuheben. Das Betriebs-Historik-Panel (BHP) basiert, wie die IABS, auf den Sozialversicherungsmeldun-

\footnotetext{
${ }^{7}$ Hier wird die Anzahl der Auszubildenden jedoch auf alle Beschäftigten und nicht nur die sozialversicherungspflichtigen Beschäftigten bezogen.

${ }^{8}$ Nähere Informationen zur Erhebung finden sich unter www.bibb.de.
} 
gen der Betriebe. Im Gegensatz zur IABS umfasst das BHP alle Betriebe mit mindestens einem sozialversicherungspflichtig Beschäftigten (Vollerhebung), es wird allerdings nur zum jährlichen Stichtag 30.6. erzeugt, weshalb Jahresdurchschnitte mit dem BHP nicht berechnet werden können. Auch das IAB-Betriebspanel wird zu einem Stichtag, dem 30.6., erhoben und beinhaltet Aussagen von Managern und Personalleitern zu unterschiedlichen betrieblichen Themen (Fischer et al. 2008). Die Ausbildungsquoten im IAB-Betriebspanel basieren somit auf den Aussagen von Managern, die möglicherweise Schätzungen vornehmen, weil ihnen aktuelle Zahlen zur beruflichen Stellung einzelner Mitarbeiter oder der Gesamtzahl der Mitarbeiter nicht vorliegen. Ein Vorteil des IAB-Betriebspanels ist, dass im Nenner nicht nur die sozialversicherungspflichtigen, sondern alle Beschäftigten der Betriebe berücksichtigt werden können. Neben diesen Datenquellen erheben das Statistische Bundesamt und das Bundesinstitut für Berufsbildung (BiBB) die Anzahl der Auszubildenden. Da für diese Datenquelle die Gesamtbeschäftigung nicht erhoben wird, werden in diesen Statistiken jedoch keine Ausbildungsquoten errechnet. ${ }^{9}$

Die Stichtage der Erhebung der Datenquellen beeinflussen die Ausbildungsquote massgeblich. Abbildung 3 verdeutlicht die Abhängigkeit der Ausbildungsquote vom Bezugszeitpunkt. Die jährliche Schwankung der Ausbildungsquote zwischen dem Ausbildungsbeginn zwischen August und Oktober und dem Ausbildungsende zwischen Februar und Juni sind deutlich erkennbar. Die Ursachen der schwankenden Ausbildungsquote liegen in der saisonalen Beschäftigung, an der vorgesehenen Dauer der Ausbildungen und an Zahl und Zeitpunkt vorzeitiger Auflösungen von Ausbildungsverträgen. ${ }^{10}$ Um die Jahreswende erreicht die Ausbildungsquote ihr Maximum und sinkt anschließend kontinuierlich bis zum 31.7. ab. Somit überschätzt der übliche Messzeitpunkt der Ausbildungsquote am 31.12. die durchschnittliche Ausbildungsquote, während sie am 30.6. unterschätzt wird. Die weiteren erkennbaren monatlichen Schwankungen sind durch die Anpassung der Gesamtbeschäftigung zu erklären, welche langsam und unterjährig variiert, während die Zahl der Auszubildenden im Prinzip nur einmal im Jahr angepasst werden kann (Abbildung 2). Aufgrund der Abhängigkeit der Ausbildungsquote vom Messzeitpunkt sollte ein Datensatz verwendet

\footnotetext{
${ }^{9}$ Die Anzahl der Auszubildenden unterscheidet sich beträchtlich zwischen der Erhebung des BiBB und der IABS. Beide Statistiken gleichen sich jedoch an, wenn die im Weiteren beschriebenen Abgrenzungen beachtet werden.

${ }^{10}$ Laut Berufsbildungsbericht 2008 werden etwa 20\% der Ausbildungsverträge vorzeitig aufgelöst, wobei die Ausbildungen etwa zur Hälfte in anderen Betrieben fortgesetzt werden. Die Ausbildungsberufe weisen dabei unterschiedliche Abbrecherquoten auf.
} 
werden, der eine Aggregation täglicher Ausbildungsquoten ermöglicht. Nur anhand der BA Beschäftigtenstatistik und der daraus entstehenden Datensätze wie der IABS können Ausbildungsquoten für jeden Tag berechnet werden. Das IAB-Betriebspanel und das BetriebsHistorik-Panel hingegen erlauben nur die Berechnung zum 30.6., die BIBB-Erhebung erfolgt zum Stichtag 30.09..

Eine weitere Ursache für Unterschiede in den ausgewiesenen Ausbildungsquoten liegt in der Abgrenzung der Auszubildenden und der Beschäftigten. Die BiBB-Daten enthalten alle Auszubildenden in anerkannten Ausbildungsberufen nach dem Berufsbildungsförderungsgesetz. Vollschulische Ausbildungen sind dabei im Gegensatz zur IABS nicht enthalten. Um eine vergleichbare Ausbildungsquote zu erhalten, sind vollschulische Auszubildende bei der Berechnung mit der IABS aus dem Zähler auszuschließen (vergleiche Abgrenzung in Grafik 4). Analog ergeben Berechnungen mit einer unterschiedlichen Abgrenzung der Beschäftigtenzahl verschiedene Quoten. So lässt sich die Ausbildungsquote in Bezug auf alle Beschäftigten oder nur auf sozialversicherungspflichtig Beschäftigte oder Vollzeitbeschäftigte beziehungsweise Vollzeitäquivalente berechnen. Die Sensitivität der Ausbildungsquote auf unterschiedliche Abgrenzungen verdeutlicht Abbildung 4. Alleine der Ausschluss Teilzeitbeschäftigter erhöht die Ausbildungsquote um einen Prozentpunkt. ${ }^{11}$

\footnotetext{
${ }^{11}$ Die Unterschiede in den wissenschaftlichen Publikationen sind natürlich außerdem auf Stichprobeneingrenzungen aufgrund des spezifischen Studiendesigns zurückzuführen.
} 


\section{Abbildung 4: Ausbildungsquoten nach unterschiedlichen Abgrenzungen von Zähler und Nenner}

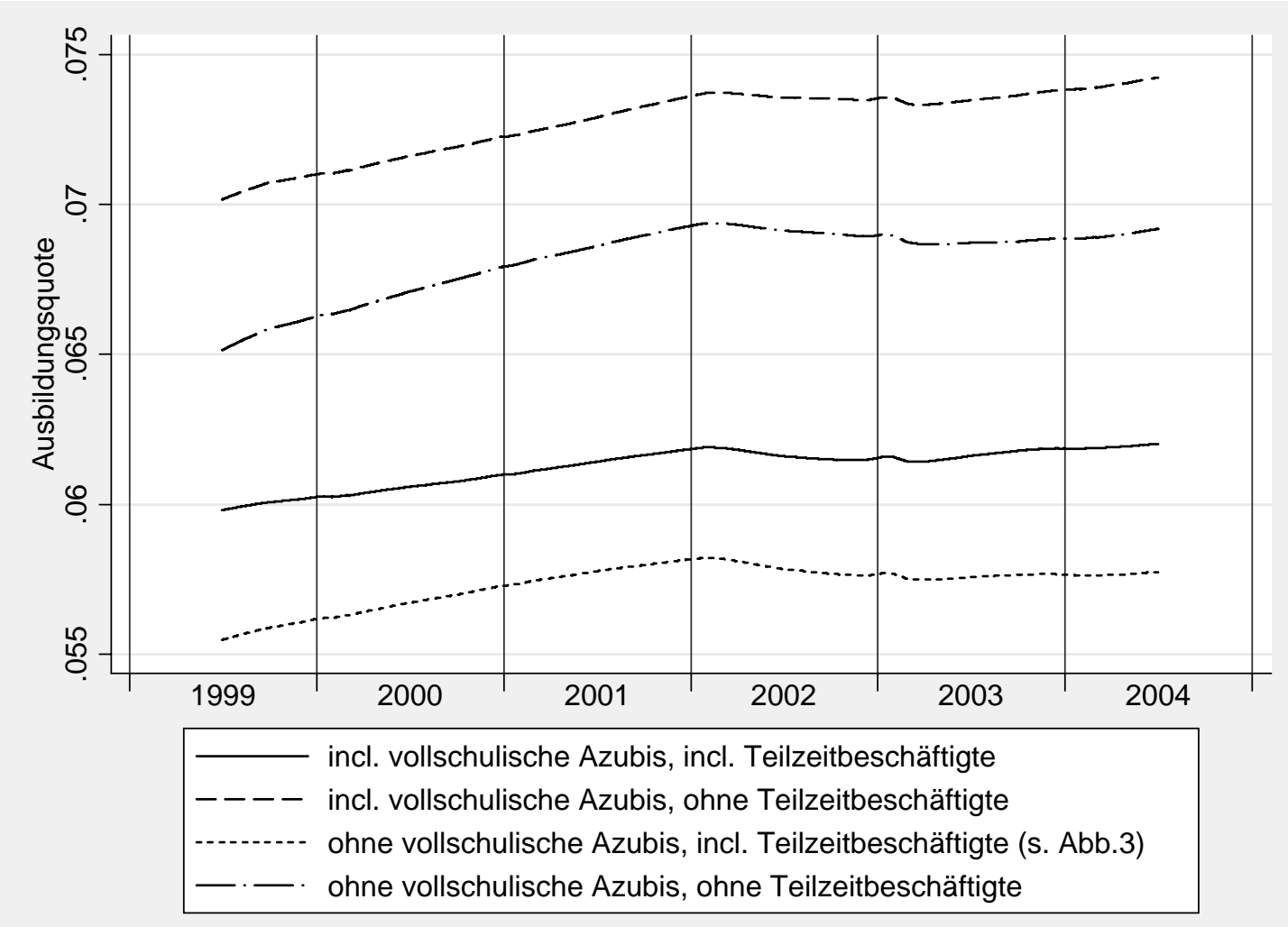

Anmerkungen: Folgende Berufe werden zu denen mit typischerweise vollschulischer Ausbildung gezählt: Heilpraktiker, Masseure, Krankengymnasten und verwandte Berufe, Krankenschwestern, -pfleger, Hebammen, Helfer in der Krankenpflege, Sozialarbeiter, Sozialpfleger, Heimleiter, Sozialpädagogen, Arbeits- und Berufsberater, Kindergärtnerinnen und Kinderpflegerinnen (Berufsklassifikationen in der IABS: 851-854 und 861-864). Ausgewiesen sind die gleitenden Jahresmittel der Ausbildungsquoten.

Quelle: IABS 1975-2004, eigene Berechnungen.

\section{Vorschlag für die Berechnung der Ausbildungsquote in Deutschland}

In diesem Kapitel schlagen wir eine Methode zur Berechnung der Ausbildungsquote in Deutschland vor. Die Ausbildungsquote weist ein starkes Saisonmuster auf (vergleiche Abbildung 3). Aufgrund unterschiedlicher Ausbildungslängen, Abbrecherquoten und nicht zuletzt unterschiedlich stark ausgeprägter Saisonabhängigkeit der Beschäftigung, sind die Saisonmuster der Ausbildungsquote berufsspezifisch. Der Stichtagsbetrachtung ist daher die Verwendung der vollen, tagesgenauen Information vorzuziehen, die eine Berechnung gleitender Jahresmittelwerte der täglichen Ausbildungsquoten wie in Abbildung 3 ermögli- 
chen. ${ }^{12}$ Sucht man eine Kennzahl pro Jahr, bietet sich der Mittelwert des Kalenderjahres an, der mit dem gleitenden Jahresmittelwert am 2. Juli übereinstimmt.

Als Datenbasis kommen für beide Berechnungsarten nur Derivate der Beschäftigtenstatistik der BA in Fragen, z.B. die IAB-Beschäftigtenstichprobe (IABS), mit der für jeden Tag des Jahres der Anteil der Auszubildenden berechnet und nach Sektorebene, Berufsebene oder für die gesamte Volkswirtschaft aggregiert ausgewiesen werden kann. Wir schlagen vor, die Auszubildenden auf der Basis des Personengruppenschlüssels 102 abzugrenzen und Personen in vollschulischer Ausbildung abzuziehen (Berufsklassifikationen in der IABS: 851-854 und 861-864). Die Anzahl der regulär sozialversicherungspflichtig Beschäftigten ergibt sich durch den Personengruppenschlüssel 101. ${ }^{13}$

Ein Nachteil dieser Berechnung liegt in der durch das Meldeverfahren zur Sozialversicherung bedingten Zeitverzögerung der Datenverfügbarkeit. ${ }^{14}$ Weiterhin sind nur sozialversicherungspflichtig Beschäftigte enthalten, was bei einzelnen Berufen mir hohem Anteil an Selbständigen oder Beamten zur Überschätzung der Ausbildungsquote führt.

\section{Vergleich saisonaler Schwankungen nach Berufsgruppen}

Anhand der zuvor dargestellten Berechnungsmethode vergleichen wir nun die Ausbildungsquoten für die drei ausgewählten Berufsgruppen der kaufmännischen Berufe, der Handwerks- und Bauberufe sowie der Produktionsberufe aus der Industrie. Diese Berufseinteilung beruht auf folgenden Unterschieden: In der Diskussion über den Ausbildungsmarkt wird der Unterschied bei der Ausbildungsmotivation zwischen Handwerk und Industrie/Dienstleistung einerseits (Franz und Soskice 1995) und zwischen kaufmännischen und produktionsbezogenen Ausbildungsberufen andererseits (Mohrenweiser und Zwick 2008) hervorgehoben. ${ }^{15}$

\footnotetext{
${ }^{12}$ Das gleitende Jahresmittelwert GJM der Ausbildungsquote AQ zum Zeitpunkt t errechnet sich wie folgt: $\mathrm{AQ}_{\mathrm{t}}^{\mathrm{GJM}}=\sum_{\mathrm{i} \in[\mathrm{t}-182, \mathrm{t}+182]} \mathrm{AQ}_{\mathrm{i}} / 365$.

${ }^{13}$ Der Personengruppenschlüssel 101 bedeutet sozialversicherungspflichtig Beschäftigte ohne besondere Merkmale. Nicht einbezogen sind Personengruppen wie Beschäftigte in Altersteilzeit, in der Seefahrt oder Werksstudenten, die aber in der Summe nur etwa 3\% der meldepflichtigen Beschäftigung ausmachen. ${ }^{14}$ Derzeit reicht die IABS bis 2004. Eine aktuellere Alternative stellt hier das BA-Beschäftigtenpanel dar, einem Quartalspanel zu den Stichtagen 31. März, 30. Juni, 30. September und 31. Dezember, derzeit bis Ende 2006 (Schmucker und Seth 2008). Nachteilig ist hier, dass für die letzten Quartale noch nicht alle Meldungen der Betriebe vorliegen und daher Fortschreibungen enthalten sind.

${ }^{15}$ Eine genaue Aufschlüsselung der einzelnen Berufe, die den Berufsgruppen zugeordnet wurden, schicken wir den interessierten Lesern gerne zu.
} 


\section{Abbildung 5: Ausbildungsquoten nach Berufsklassen 2004}

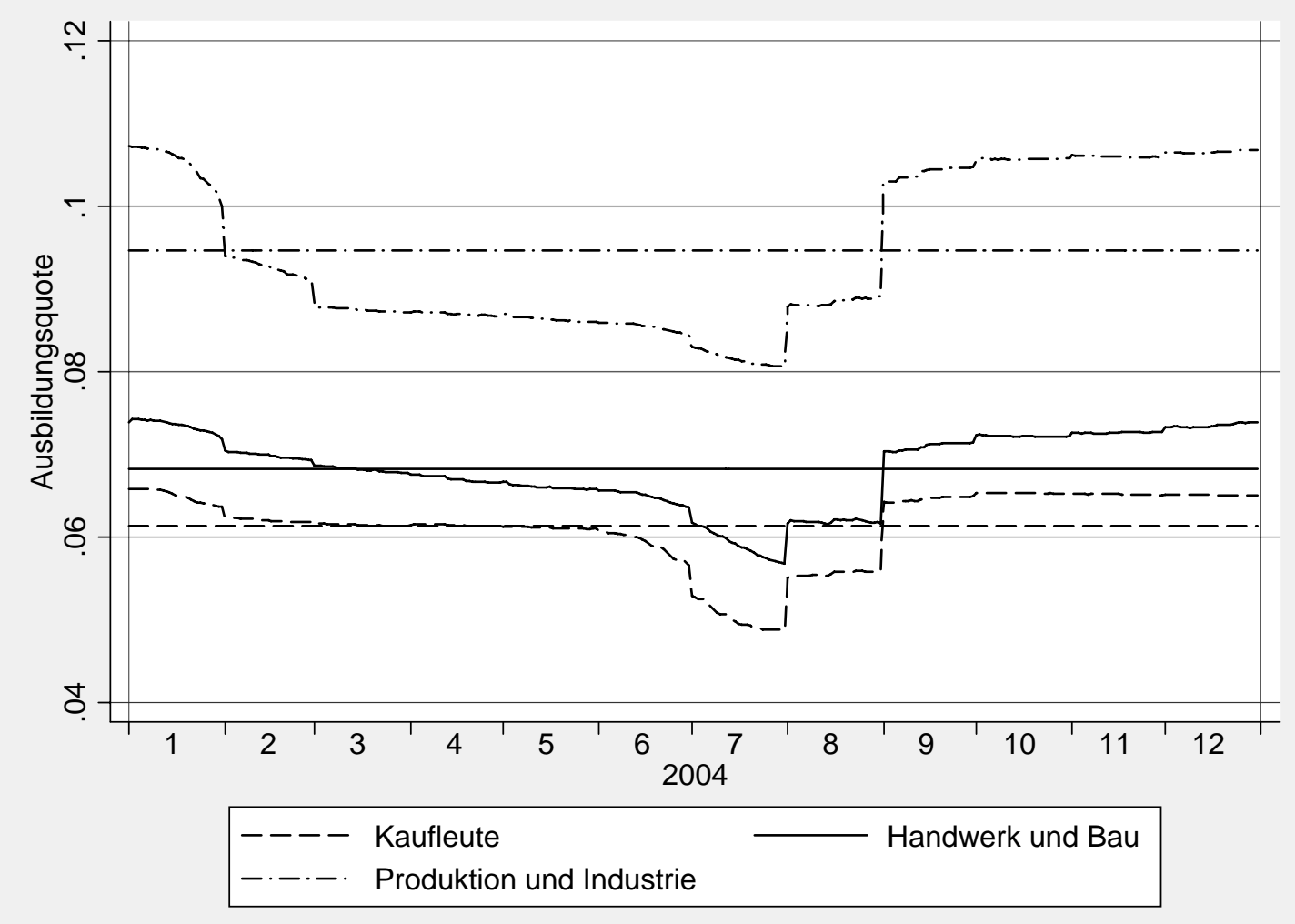

Anmerkungen: Die waagrechten Linien sind Jahresmittelwerte.

Quelle: IABS 1975-2004, eigene Berechnungen.

Ein Vergleich der saisonalen Schwankungen in den einzelnen Berufsgruppen fördert die erwarteten Muster zutage (siehe Abbildung 5). Ein Großteil der kaufmännischen Ausbildungsgänge ist dreijährig. Dadurch bleibt die Ausbildungsquote während des ersten Halbjahres relativ konstant und sinkt von Mai bis Ende Juli. In den Produktionsberufen im industriellen Bereich überwiegen hingegen die dreieinhalbjährigen Ausbildungsgänge. Deshalb sinkt hier die Ausbildungsquote bereits am 1.2. und am 1.3. jeden Jahres und bleibt dann bis zum Beginn des neuen Ausbildungsjahres konstant niedrig. Im Handwerk sinkt die Ausbildungsquote schließlich fast kontinuierlich ab dem 1.2. bis zum 31.7., da es hier sowohl drei- als auch dreieinhalbjährige Ausbildungen gibt, vor allem aber saisonbedingt die Beschäftigung steigt. Abbildung 7 im Anhang zeigt das gleiche Muster für die Jahre 1999 bis 2003. Dies verdeutlicht nochmals, dass aus einer Stichtagfestlegung unterschiedliche und relativ willkürliche berufsbezogene Ausbildungsquoten resultieren, die den jeweiligen Ausbildungsanstrengungen der Unternehmen in den Ausbildungsberufen nicht gerecht werden. 


\section{Schlussfolgerungen}

Dieser Beitrag schlägt eine neue Methode zur Berechnung durchschnittlicher Ausbildungsquoten in Deutschland vor. Hierbei werden tagesgenaue Ausbildungsquoten aus der IABBeschäftigtenstichprobe berechnet und aggregiert. Die Relevanz der Berechnung durchschnittlicher Ausbildungsquoten aus tagesgenauen Statistiken ergibt sich aufgrund des saisonalen Musters der Eintritte in die Ausbildung und der typischen Zeiten des Ausbildungsendes. Die auf das Kalenderjahr bezogenen durchschnittlichen Ausbildungsquoten lagen in den Jahren 1999 bis 2004 zwischen 5,55\% und 5,78\%. Das Minimum lag hierbei jeweils einen Prozentpunkt darunter, das Maximum 0,5 Prozentpunkte darüber. Messungen der Ausbildungsquote zu Zeitpunkten, die nahe am Minimum liegen wie der Berufsbildungsbericht am 30.6. oder am Maximum wie der Bericht „Bildung in Deutschland“ am 31.12., sind Momentaufnahmen an besonderen Tagen, die die Ausbildungsbereitschaft der Unternehmen im betreffenden Jahr über- bzw. unterzeichnen. Der unterschiedlichen Ausbildungslänge in den verschiedenen Ausbildungsberufen und den damit verknüpften unterschiedlichen saisonalen Mustern wird eine willkürliche Stichtagfestlegung nicht gerecht. Dies bedeutet wiederum, dass an bestimmten Stichtagen die Abweichung von der durchschnittlichen Ausbildungsquote von Berufsgruppe zu Berufsgruppe schwankt. 


\section{Literatur}

Autorengruppe Bildungsberichterstattung (2008): Bildung in Deutschland 2008, Bertelsmann, Bielefeld.

Beckmann, M. (2002), Firm-sponsored Apprenticeship Training in Germany: Empirical Evidence from Establishment Data, Labour 16(2), S. 289-310.

Bundesministerium für Bildung und Forschung (2007), Berufsbildungsbericht 2006, Berlin.

Bundesministerium für Bildung und Forschung (2008), Berufsbildungsbericht 2008, Berlin.

Statistik der Bundesagentur für Arbeit (2007), Arbeitsmarkt in Zahlen, Ausbildungsstellenmarkt - Bewerber und Berufsausbildungsstellen - 2007

Dietrich, H., K. Dressel, F. Janik, W. Ludwig-Mayerhofer (2008): Ausbildung im dualen System und Maßnahmen der Berufsvorbereitung IAB-Handbuch, Kapitel 5, Nürnberg.

Drews, N. (2007), Variablen der schwach anonymisierten Version der IAB-BeschäftigtenStichprobe 1975-2004, FDZ Datenreport 03/2007, Nürnberg.

Dustmann, C. und U. Schönberg (2004), Training and Union Wages, IZA Discussion Paper 1435, Bonn.

Fischer, G., F. Janik, D. Müller und A. Schmucker (2008), Das IAB-Betriebspanel - von der Stichprobe über die Erhebung bis zur Hochrechnung, FDZ Methodenreport 1/2008, Nürnberg.

Franz, W. und D. Soskice (1995), The German Apprenticeship System, in F. Buttler, W. Franz, R. Schettkat and D. Soskice (Eds.): Institutional Frameworks and Labor Market Performance, Routledge, London, pp. 208-34.

Institut der deutschen Wirtschaft (2007): Deutschland in Zahlen, Köln.

Konsortium Bildungsberichterstattung (2006): Bildung in Deutschland, Bertelsmann, Bielefeld.

Mohrenweiser, J. und T. Zwick (2008): Why Do Firms Train Apprentices? The Net Cost Puzzle Reconsidered, ZEW Discussion Paper No. 08-019, Mannheim.

Schmucker, A. und S. Seth (2008), BA-Beschäftigtenpanel 1998-2006, Codebuch, FDZ Datenreport 01/2008, Nürnberg.

Spengler, A. (2007), Das Betriebs-Historik-Panel 1975-2005, FDZ Datenreport 04/2007, Nürnberg. 
Statistik der Bundesagentur für Arbeit (2007), Tabelle 4.1 „Bestand an unbesetzten Berufsausbildungsstellen und noch nicht vermittelten Bewerbern für Berufsausbildungsstellen“. http://www.pub.arbeitsamt.de/hst/services/statistik/detail_2004/p.html,

Statistisches Bundesamt (2008), Statistik der allgemeinbildenden Schulen, Zeitreihen aus 21111LJ002, GENESIS-Online, Stand: 04.06.2008.

Ulrich, J., B. Ehrenthal; E. Krekel (2004), Durchwachsene Bilanz in der Ausbildungsvermittlungsstatistik, Bildungspraxis. Zeitschrift für die technische Aus- und Weiterbildung 4, S. 10-13.

Ulrich J., S. Flemming, R. Granath, E. Krekel (2006), Zahl der neu abgeschlossenen Ausbildungsverträge fällt auf den niedrigsten Stand seit der Wiedervereinigung, Informationen für die Bildungs- und Berufsberatung 16, S. 1-37.

Wachter, T. (2008), Personalökonomische Implikationen zur Gestaltung von Anreizstrukturen in der betrieblichen Berufsausbildung, Dissertation Ludwig-MaximiliansUniversität, München. 


\section{Appendix}

\section{Abbildung 6: Gesamtwirtschaftliche Ausbildungsquote und durchschnittliche betrieb- liche Ausbildungsquote}

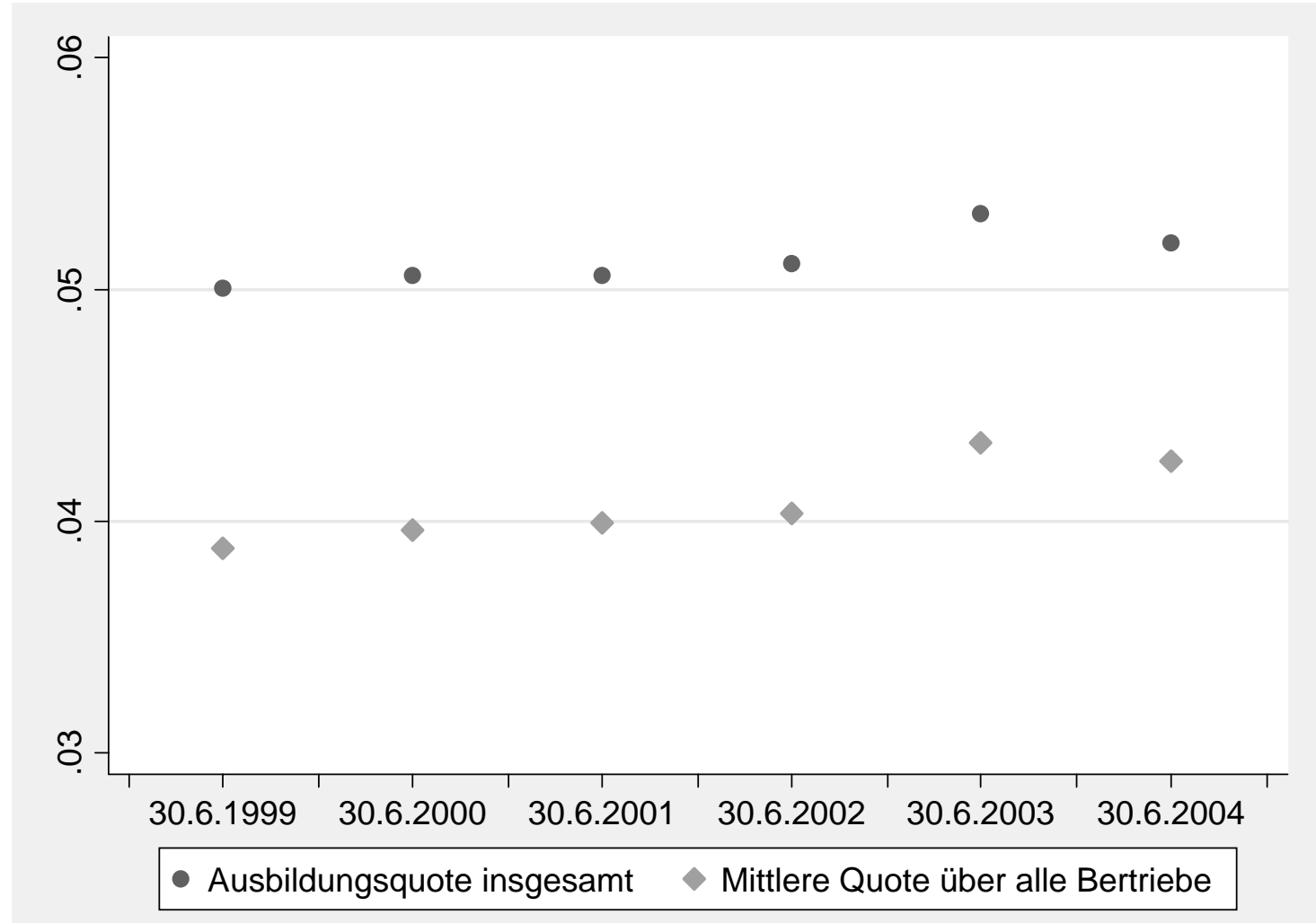

Anmerkung: Die Ausbildungsquoten auf Basis des Betriebs-Historik-Panels (BHP) stimmen weitgehend mit den Werten auf Basis der IABS überein (siehe Abbildung 3, Reihe Stichtag 30.6.). Kleine Abweichungen ergeben sich zum einen dadurch, dass es sich bei der IABS um eine 2\%-Stichprobe handelt und beim BHP um die Grundgesamtheit aller Betriebe mit mindestens einem sozialversicherungspflichtig Beschäftigten. Zum anderen enthält die Zahl der Beschäftigten aus dem BHP auch die kleine Gruppe sozialversicherungspflichtig Beschäftigter mit besonderen Merkmalen, wie z.B. Beschäftigte in Altersteilzeit oder Werksstudenten. Der größere Nenner führt entsprechend zu einer etwas geringeren Ausbildungsquote.

Quelle: Betriebs-Historik-Panel (Spengler, 2007), eigene Berechnungen. 
Abbildung 7: Ausbildungsquoten nach Berufsklassen 1999-2004

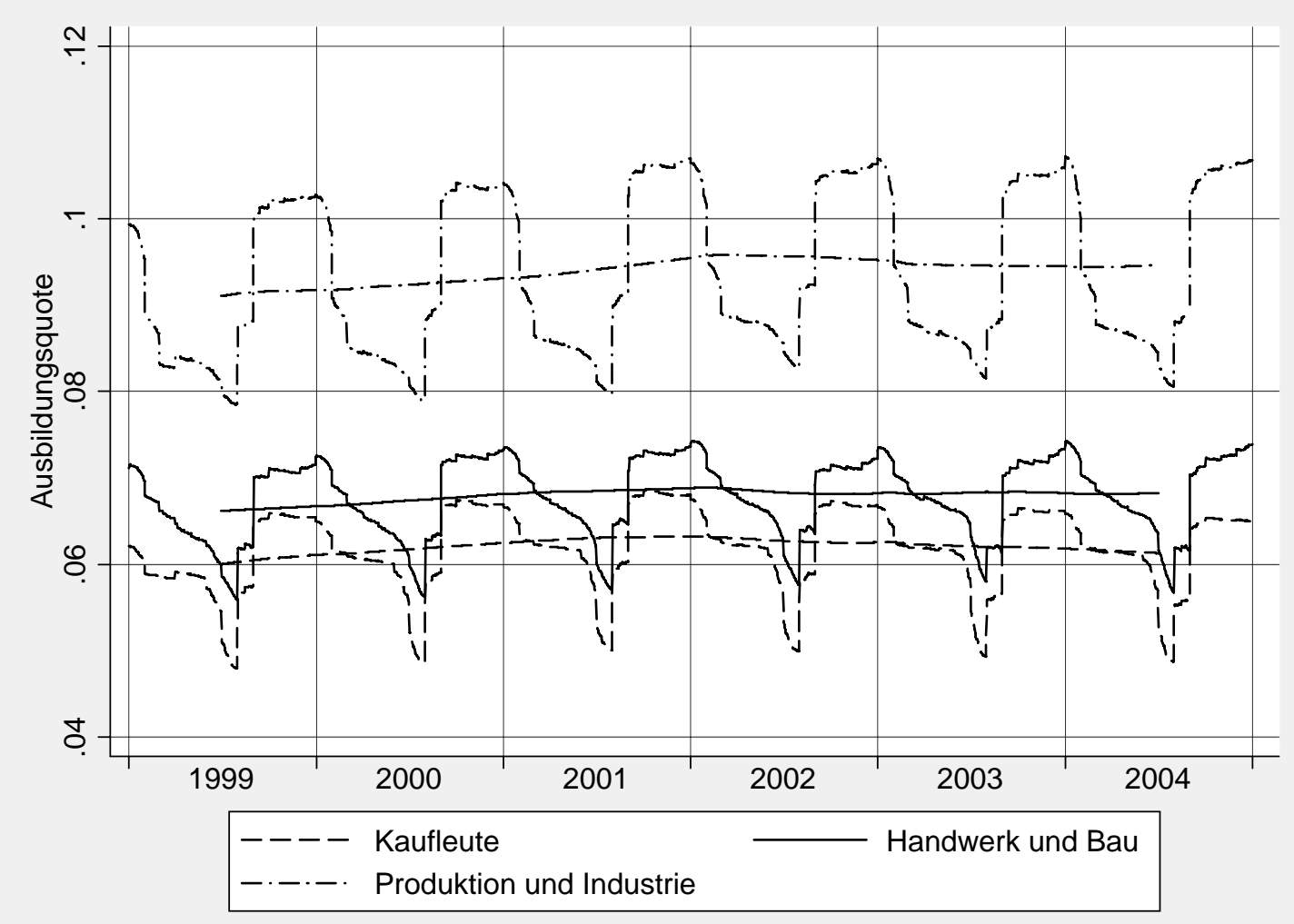

Anmerkungen: Die geglätteten Kurven sind gleitende Jahresmittel.

Quelle: IABS 1975-2004, eigene Berechnungen. 